\title{
Dynamics of inhibition patterns during fermentation processes-Zea Mays and Sorghum Bicolor case study
}

\author{
Neba F. Abunde ${ }^{1}$ N. Asiedu ${ }^{2}$ Ahmad Addo ${ }^{1}$
}

Received: 15 January 2016/ Accepted: 7 November 2016/Published online: 15 November 2016

(c) The Author(s) 2016. This article is published with open access at Springerlink.com

\begin{abstract}
Recently ethanol production involved the processing and fermentation of sorghum and maize extracts. Sorghum and maize are cheaper, locally available and a substitute to imported barley malt. Large scale ethanol fermentation systems are usually hampered by instability, in the form of oscillations resulting from ethanol inhibition and the lag response of yeast cells to this inhibition. There is limited information regarding the mathematical nature of such inhibitions in the fermentation of sorghum and maize extracts. In the present work, mathematical models are developed to determine the nature of ethanol inhibition during the fermentation of sorghum and maize extracts. The models were sets of coupled ordinary differential equations based on a Monod type cell growth kinetic model that accounts for product inhibition. The Inhibition patterns considered were; Linear, Sudden Growth Stop and Exponential. The results obtained showed that there is product inhibition during ethanol fermentation using sorghum extracts, with inhibition patterns being Linear and Exponential. However, the results obtained from ethanol fermentation of maize extract also showed that there is product inhibition during ethanol fermentation using maize extracts, with inhibition patterns being Linear and Sudden Growth Stop. The obtained models described with high accuracy, 99\% Confidence Interval the dynamics of
\end{abstract}

N. Asiedu

nasiedusoe@yahoo.co.uk

1 Department of Agricultural Engineering, College of Engineering, Kwame Nkrumah University of Science and Technology, Kumasi, Ghana

2 Department of Chemical Engineering, College of Engineering, Kwame Nkrumah University of Science and Technology, Kumasi, Ghana substrate utilization, product formation and cell growth. These inhibitions which affect the high ethanol yields can be minimized by setting up an optimal control problem using the developed models and solved to determine the control variables that minimize the effect of such inhibitions during the fermentation of sorghum and maize extracts.

Keywords Alcoholic fermentation - Mathematical modeling $\cdot$ Ethanol inhibition · Maize extracts sorghum extracts

\section{List of symbols}

$\mu_{\max } \quad$ Maximum specific growth rate $\left(\mathrm{h}^{-1}\right)$

$q_{\text {pmax }}$ Maximum rate of product formation $\left(\mathrm{h}^{-1}\right)$

$P_{\text {xmax }}$ Product concentration when product formation ceases $(\mathrm{g} / 100 \mathrm{~g})$

$P_{\text {pmax }}$ Product concentration when cell growth ceases (g/ $100 \mathrm{~g})$

$K_{\text {ix }} \quad$ Product inhibition coefficient on cell growth

$K_{\text {ip }} \quad$ Product inhibition coefficient on product formation

$K_{\text {isx }} \quad$ Substrate inhibition coefficient on cell growth

$K_{i s p} \quad$ Substrate inhibition coefficient on product formation

$K_{\text {sx }} \quad$ Substrate saturation (Monod) constant for cell growth $(\mathrm{g} / 100 \mathrm{~g})$

$K_{\text {sp }} \quad$ Substrate saturation (Monod) constant for product formation $(\mathrm{g} / 100 \mathrm{~g})$

$Y_{\mathrm{x}} \quad$ Yield coefficient of cell based on substrate utilization $(\mathrm{g} / \mathrm{g})$

$Y_{\mathrm{p}} \quad$ Yield coefficient of cell based on substrate utilization $(\mathrm{g} / \mathrm{g})$

$G_{\text {s }} \quad$ Yield coefficient of cell based on substrate utilization $(\mathrm{g} / \mathrm{g}$ h) 
$M_{\mathrm{s}} \quad$ Cell growth coefficient on substrate $(\mathrm{g} / \mathrm{g} \mathrm{h})$

\section{Introduction}

In several studies regarding the alcoholic fermentation oscillations in batch fermenters resulting from ethanol inhibition and the lag response to yeast cells to this inhibition has been observed and reported. It is often conventional during the modeling of ethanol fermentation to predefine an inhibition pattern but the success of this practice is based on probabilities, since such patterns vary based on the type and strength of the fermentation wort. Sorghum, a cereal which belongs to the family Graminae was first used as a brewing adjunct during the Second World War and is now used in most breweries as locally available alternative to imported barley malt. In recent years, the search for cheaper locally available substitutes to imported barley malt rekindled the involvement of most firms in expensive experiments regarding beer production from various materials and today, most of the more successful firms use maize and sorghum in their beer production process. In a generalized view of processing sorghum and maize for beer production, though involves several unit operations, the fermentation step is regarded as the heart of the entire production where a near optimal environment is desired for microorganisms to grow, multiply and produce the desired product, Alford [1]. However, the fermentation of sorghum and maize extracts at large scale is usually hampered by sub optimal conditions including instability, in the form of oscillations resulting from ethanol inhibition and the lag response of yeast cells to this inhibition, Chen and McDonald [2, 3], Beuse et al. [4], Fengwu [5]. These inhibitions observed results in an increase in residual sugar at the end of the fermentation, which decreases raw material consumption and correspondingly, decreases the ethanol yield if no economically acceptable attenuation strategies are developed, Fengwu [5]. In a typical procedure for modeling ethanol fermentation, if inhibition is considered, it is often conventional to predefine the inhibition pattern and this practice increases uncertainties in the model since ethanol inhibition pattern varies depending on the type of microorganism, and on the type and strength of fermentation wort, Russell [6]. This increase the unreliability of process controllers and simulators since these automatic tools are usually based on a mathematical representation of the considered system, Alford [1]. Dynamic models were developed, incorporating three effects of product inhibition into the kinetic model and simulation resulted in interesting findings.

\section{Model development}

The fermentation process kinetics was described with a Monod type cell growth model that accounts for substrate and product inhibition.

\section{Modeling kinetics of growth and product formation}

Starting from the Monod Equation for cell growth and product formation, Eq. (1), three inhibition patterns were considered in modeling product inhibition; linear, Sudden growth stop and exponential as shown in Table 1 below.

$\mu(S)=\frac{\mu_{\max } S}{K_{s x}+S}$

$\boldsymbol{q}_{p}(S)=\frac{\boldsymbol{q}_{p \max } S}{\boldsymbol{K}_{\boldsymbol{s p}}+\boldsymbol{S}}$

Introducing the effect of product inhibition on the Monod equation, using the respective inhibition factors, the following kinetic models were obtained:

Kinetics with Linear Product Inhibition, -HinshelwoodDagley model [7]

$\boldsymbol{\mu}(\boldsymbol{S}, \boldsymbol{P})=\left(1-\boldsymbol{K}_{i x} \boldsymbol{P}\right) \frac{\boldsymbol{\mu}_{\max } \boldsymbol{S}}{\boldsymbol{K}_{\boldsymbol{s} x}+\boldsymbol{S}}$

$\boldsymbol{q}(\boldsymbol{S}, \boldsymbol{P})=\left(1-\boldsymbol{K}_{i p} \boldsymbol{P}\right) \frac{\boldsymbol{q}_{\max } \boldsymbol{S}}{\boldsymbol{K}_{s x}+\boldsymbol{S}}$

Kinetics Sudden Growth Stop Product Inhibition, Ghose and Tyagi [8]

$\boldsymbol{\mu}(\boldsymbol{S}, \boldsymbol{P})=\left(1-\frac{\boldsymbol{P}}{\boldsymbol{P}_{\max }}\right) \frac{\boldsymbol{\mu}_{\max } S}{\boldsymbol{K}_{s x}+\boldsymbol{S}}$

$\boldsymbol{q}(\boldsymbol{S}, \boldsymbol{P})=\left(1-\frac{\boldsymbol{P}}{\boldsymbol{P}_{\text {pmax }}}\right) \frac{\boldsymbol{q}_{\max } S}{K_{s x}+S}$

Kinetics with Exponential Product Inhibition,- Aiba and Shoda model [9]

$\mu(\boldsymbol{S}, \boldsymbol{P})=\exp \left(-\boldsymbol{K}_{i x} \boldsymbol{P}\right) \frac{\boldsymbol{\mu}_{\max } \boldsymbol{S}}{\boldsymbol{K}_{\text {sx }}+\boldsymbol{S}}$
$q(\boldsymbol{S}, \boldsymbol{P})=\exp \left(-\boldsymbol{K}_{i p} \boldsymbol{P}\right) \frac{\boldsymbol{q}_{\max } \boldsymbol{S}}{\boldsymbol{K}_{s x}+\boldsymbol{S}}$

Table 1 Mathematical expressions for the product inhibition factors

\begin{tabular}{ll}
\hline Product inhibition factor & Mathematical expression \\
\hline Linear & $\left(1-K_{2} P\right)$ \\
Sudden growth stop & $\left(1-\frac{P}{P_{\max }}\right)$ \\
Exponential & $e^{-K_{1} P}$ \\
\hline
\end{tabular}




\section{Material Balance and development of Differential Equations}

The dynamic equations describing the cell growth, product formation and substrate utilization can be developed by applying the principle of conservation of mass. The following system of first order ordinary differential equations that present cell growth, product formation and substrate utilization are in Eqs. (9)-(11)

$\frac{\mathrm{d} X}{\mathrm{~d} t}=\mu X$

$\frac{\mathrm{d} P}{\mathrm{~d} t}=q X$

$\frac{\mathrm{d} S}{\mathrm{~d} t}=-\frac{1}{Y_{x}} \frac{\mathrm{d} X}{\mathrm{~d} t}-\frac{1}{Y_{p}} \frac{\mathrm{d} P}{\mathrm{~d} t}-G_{s} X-M_{s} X$

Using the batch kinetic models developed above and substituting $\mu$ and $q$ in Eq. (5) with each of their product inhibition expressions, the approximate representation of the fermentation process in each inhibition scenario could be described by the following equations;

Dynamics with linear product inhibition:

$$
\begin{aligned}
\frac{\mathrm{d} X}{\mathrm{~d} t} & =\left(1-K_{i x} P\right) \frac{\mu_{\max } S}{K_{s x}+S} X \\
\frac{\mathrm{d} P}{\mathrm{~d} t} & =\left(1-K_{i p} P\right) \frac{q_{\max } S}{K_{s p}+S} X \\
\frac{\mathrm{d} S}{\mathrm{~d} t} & =-\frac{1}{Y_{x}} \frac{\mathrm{d} X}{\mathrm{~d} t}-\frac{1}{Y_{p}} \frac{\mathrm{d} P}{\mathrm{~d} t}-G_{S} X-M_{s} X
\end{aligned}
$$

Dynamics with sudden growth stop product inhibition:

$$
\begin{aligned}
\frac{\mathrm{d} X}{\mathrm{~d} t} & =\left(1-\frac{P}{P_{\max }}\right) \frac{\mu_{\max } S}{K_{s x}+S} X \\
\frac{\mathrm{d} P}{\mathrm{~d} t} & =\left(1-\frac{P}{P_{p \max }}\right) \frac{q_{\max } S}{K_{s p}+S} X \\
\frac{\mathrm{d} S}{\mathrm{~d} t} & =-\frac{1}{Y_{x}} \frac{\mathrm{d} X}{\mathrm{~d} t}-\frac{1}{Y_{p}} \frac{\mathrm{d} P}{\mathrm{~d} t}-G_{s} X-M_{s} X
\end{aligned}
$$

Dynamics with exponential product inhibition:

$$
\begin{aligned}
\frac{\mathrm{d} X}{\mathrm{~d} t} & =\exp \left(-K_{i x} P\right) \frac{\mu_{\max } S}{K_{s x}+S} X \\
\frac{\mathrm{d} P}{\mathrm{~d} t} & =\exp \left(-K_{i p} P\right) \frac{q_{\max } S}{K_{s p}+S} X \\
\frac{\mathrm{d} S}{\mathrm{~d} t} & =-\frac{1}{Y_{x}} \frac{\mathrm{d} X}{\mathrm{~d} t}-\frac{1}{Y_{p}} \frac{\mathrm{d} P}{\mathrm{~d} t}-G_{S} X-M_{S} X
\end{aligned}
$$

\section{Parameter Estimation and Model Statistical Validity}

The identification of model parameters for each of the three systems of equations was made with Matlab and the ode45 solver used to simulate the differential equations. This was done by minimizing the overall sum of squared error, Eq. (11) between the model simulation and experimental data points of the process variables (Biomass, Substrate and Product).

$$
\begin{array}{r}
\varepsilon=\min \sum\left(X\left(k_{1}, k_{2}, \ldots, k_{n}\right)-X^{e}\right)^{2} \\
+\left(S\left(k_{1}, k_{2}, \ldots, k_{n}\right)-S^{e}\right)^{2} \\
+\left(P\left(k_{1}, k_{2}, \ldots, k_{n}\right)-P^{e}\right)^{2}
\end{array}
$$

For that purpose the Matlab routine "fmincon" was applied. Here $k_{i}, i=1 \div n$ was vector of model parameters to be determined as output of minimization procedure. Once the model parameters were estimated, the capability of the mathematical model to describe the ethanol fermentation process was tested statistically using the F- tests and this was done using STATA at a confidence interval of $99 \%$, to find out the confidence level for the developed mathematical model.

\section{Results and discussion of sorghum extract}

Table 2 presents the parameters for the four different

\begin{tabular}{|c|c|c|c|c|}
\hline & \multicolumn{4}{|l|}{ Inhibition } \\
\hline & No Inhibition & Linear & SGS & Exponential \\
\hline \multicolumn{5}{|c|}{ Model parameters } \\
\hline$\mu_{\max }$ & 0.3887 & 0.9557 & 0.6582 & 2.2628 \\
\hline$q_{\text {pmax }}$ & 17.4649 & 2.2101 & 4.7227 & 9.0145 \\
\hline$P_{x \max }$ & & & 7.9885 & \\
\hline$P_{p m a x}$ & & & 9.8916 & \\
\hline$K_{i x}$ & & 0.1294 & & 0.4210 \\
\hline$K_{i p}$ & & 0.1004 & & 0.1030 \\
\hline$K_{s x}$ & 249.9922 & 125.433 & 81.8551 & 179.2911 \\
\hline$K_{s p}$ & 199.9980 & 29.213 & 74.8407 & 199.8710 \\
\hline$Y_{x}$ & 0.1001 & 0.1086 & 0.1368 & 1.0000 \\
\hline$Y_{p}$ & 0.6085 & 1.8895 & 1.2710 & 0.5936 \\
\hline$G_{s}$ & 0.0010 & 0.0502 & 0.0345 & 0.0010 \\
\hline$M_{s}$ & 0.0100 & 0.0564 & 0.0423 & 0.0100 \\
\hline Model error & 5.4262 & 0.3407 & 0.4054 & 0.3270 \\
\hline
\end{tabular}
models used to describe the dynamics of fermentation, while Figs. 1, 2, 3 and 4 presents the fitting of the models with respect to the experimental data. The results led to the following interesting conclusions; alcoholic fermentation of sorghum extracts using Saccharomyces cerevisiae shows the existence of product inhibition, and the patterns of inhibition could be described as linear or exponential models which showed lowest error. It is shown in this work that the errors for the models showing product inhibition

Table 2 Model Parameters for fermentation using Sorghum Extracts 


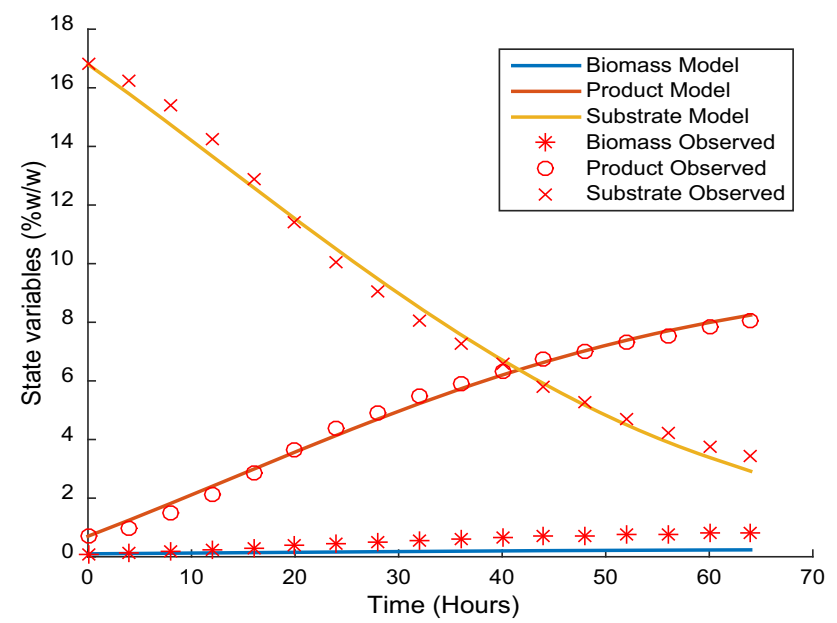

Fig. 1 Experimental results and model fitting, case of no inhibition (Monod-model)

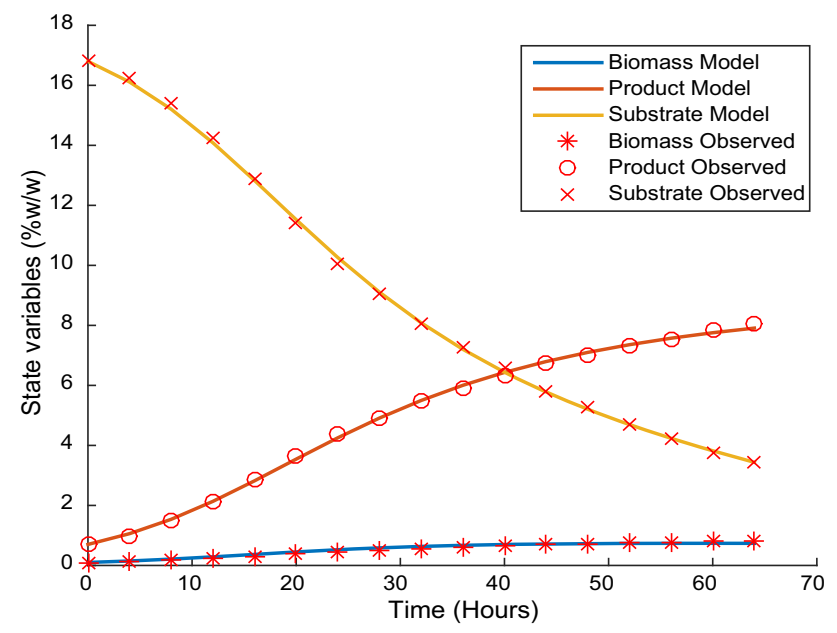

Fig. 2 Experimental results and model fitting, case of linear inhibition model

were all lower than that of the Monod which was used as a control. The results confirmed previous observations by Chen and McDonald, 1990a,b; Beuse et al., 1998, 1999; Ingledew, 1999 and Fengwu, 2007 that results ethanol, whether produced by yeast cells during fermentation or externally added into a fermentation system, can trigger inhibitions once its concentration approaches inhibitory levels. Also important in fermentation kinetics is the product yield, growth and maintenance coefficients.

The linear model showed a relatively high product yield coefficient with a relatively low growth and maintenance coefficient compared to the exponential model. This suggest that even though both models showed similar accuracy in describing the fermentation dynamics, designing a control policy with the Linear model will result in more substrate being converted into extracellular product and lesser amount for cell growth and maintenance hence

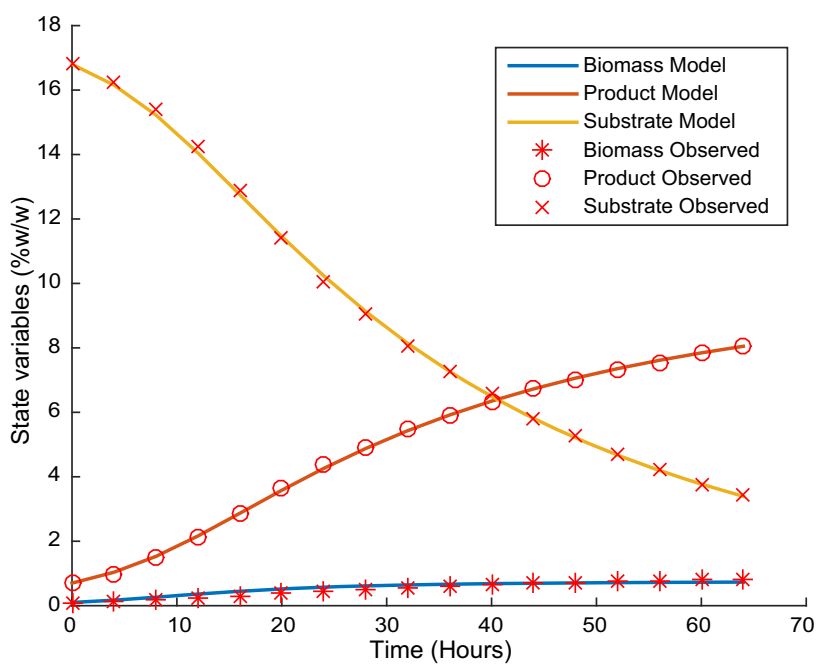

Fig. 3 Experimental results and model fitting, case of Exponential inhibition model

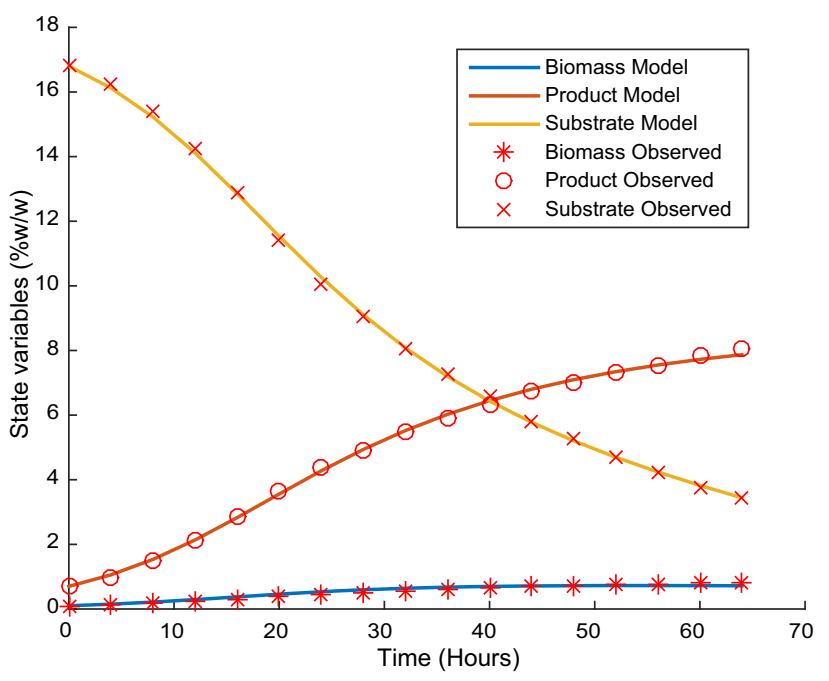

Fig. 4 Experimental results and model fitting, case of Sudden Growth Stop inhibition model

higher productivity and yield. Tables 3 and 4 presents the model statistical validity using two sample F-test for variance and the results show that at a $99 \%$ confidence interval, the states prediction of the linear and exponential models showed no significant difference with the experimental data.

Also important in a fermentation process is how the substrate and product vary in the fermenter as the cells grow. The linear and exponential inhibition models were both used to simulate this variation and both showed a decrease in substrate concentration and an increase in product concentration with cell growth. However, with the linear model the process arrives at steady state within the time used for the simulation as shown in Fig. 5 by the curve at the edges of the plots. This behavior was not 
Table 3 Model Statistical Validity with kinetics of linear inhibition, two sample F-test for variance (Biomass)

\begin{tabular}{|c|c|c|c|c|c|c|}
\hline & \multicolumn{2}{|l|}{ Biomass } & \multicolumn{2}{|l|}{ Product } & \multicolumn{2}{|l|}{ Substrate } \\
\hline & Experimental (Xobs) & Model (Xpred) & Experimental (Pobs) & Model (Ppred) & Experimental (Sobs) & Model (Spred) \\
\hline Mean & 0.508 & 0.533 & 4.903 & 4.893 & 9.126 & 9.107 \\
\hline Standard Error & 0.059 & 0.055 & 0.610 & 0.605 & 1.114 & 1.105 \\
\hline Standard Deviation & 0.244 & 0.228 & 2.514 & 2.495 & 4.595 & 4.558 \\
\hline Observations & 17 & 17 & 17 & 17 & 17 & 17 \\
\hline Confidence Interval & 0.990 & & 0.990 & & 0.990 & \\
\hline$F$ & 0.8664 & & 0.9842 & & 0.9840 & \\
\hline $\operatorname{Pr}(F<f)$ Two-tailed & 0.7778 & & 0.9750 & & 0.9747 & \\
\hline
\end{tabular}

Table 4 Model Statistical Validity with kinetics of Exponential inhibition, two sample F-test for variance

\begin{tabular}{|c|c|c|c|c|c|c|}
\hline & \multicolumn{2}{|l|}{ Biomass } & \multicolumn{2}{|l|}{ Product } & \multicolumn{2}{|l|}{ Substrate } \\
\hline & Experimental (Xobs) & Model (Xpred) & Experimental (Pobs) & Model (Ppred) & Experimental (Sobs) & Model (Spred) \\
\hline Mean & 0.508 & 0.549 & 4.903 & 4.903 & 9.126 & 9.105 \\
\hline Standard error & 0.059 & 0.050 & 0.610 & 0.610 & 1.114 & 1.1061 \\
\hline Standard deviation & 0.244 & 0.207 & 2.514 & 2.514 & 4.595 & 4.561 \\
\hline Observations & 17 & 17 & 17 & 17 & 17 & 17 \\
\hline Confidence interval & 0.990 & & 0.990 & & 0.990 & \\
\hline$F$ & 0.7209 & & 0.9994 & & 0.9850 & \\
\hline
\end{tabular}

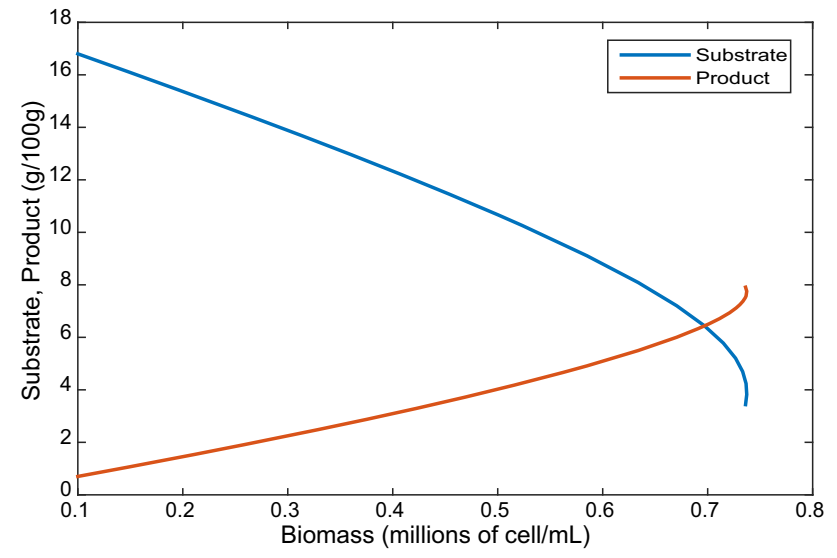

Fig. 5 Simulation of substrate and Product variation as a function of biomass during fermentation using linear inhibition kinetics

observed in the exponential inhibition model as shown in Fig. 6, confirming our earlier assertions with the yield coefficients that using the linear model in a control policy will result in high productivity. 3D profiles using the proximal interpolant method implemented using the Matlab curve fitting tool also revealed interesting findings regarding the fermentation process. This was to observe the formation of product as cells grow and consume substrate.

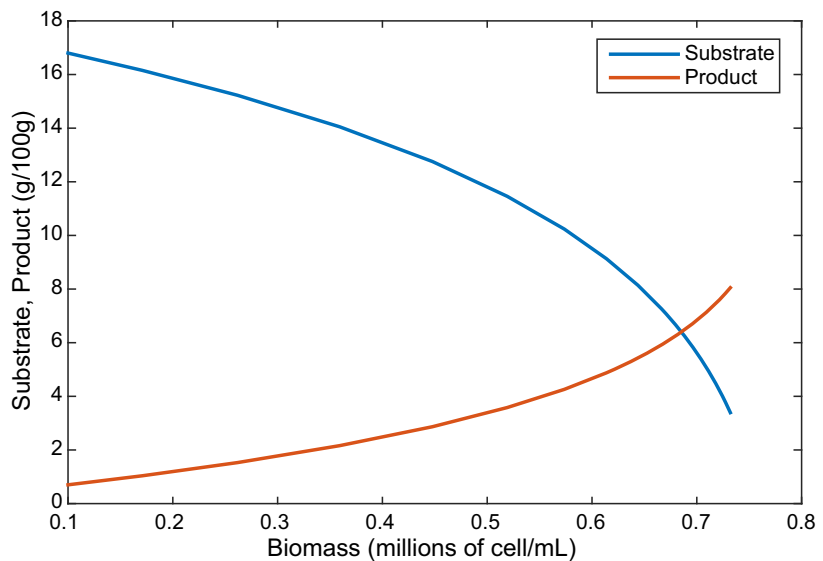

Fig. 6 Simulation of substrate and Product as a function of biomass during fermentation using exponential inhibition kinetics

Figure 7 presents product variation with cell growth and substrate consumption using the linear model and Fig. 8 using the exponential model. It can be found that with the linear model, as ethanol accumulated in the fermenter up to a certain concentration, nonlinearities, described as instabilities were observed in the product profile. This can be attributed to the higher product yield coefficient of the linear model compared to the exponential model which 


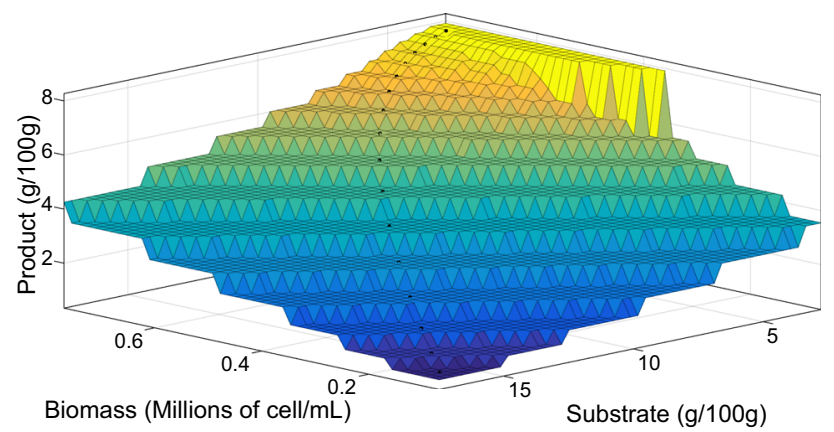

Fig. 7 3D Proximal Interpolant Simulation of Product variation as a function of substrate and biomass using Linear inhibition kinetic Model

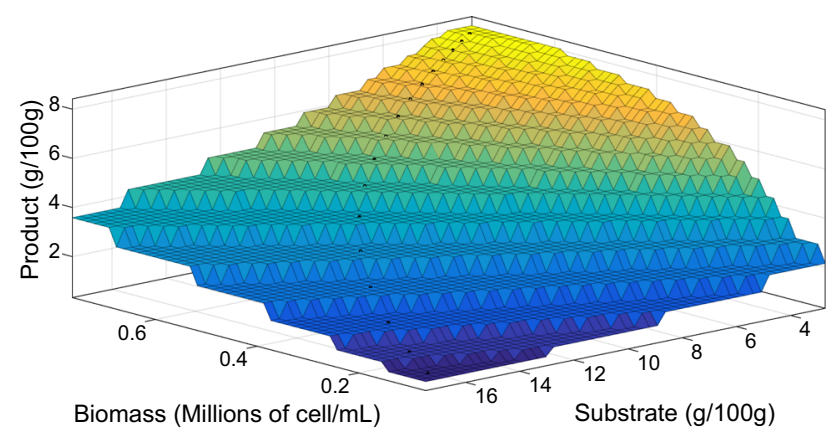

Fig. 8 3D Proximal Interpolant Simulation of Product variation as a function of substrate and biomass using Exponential inhibition kinetic Model

resulted in ethanol accumulating faster in the fermenter and rapidly reaching inhibitory levels, resulting in transient instabilities in the fermenter showed by the nonlinearity, Ingledew [10], Fengwu [5]. This high ethanol concentration is inhibitory to the yeast cell by disrupting the integrity of the cell membrane, Russell [6], Sutton [11]. Figures 9,

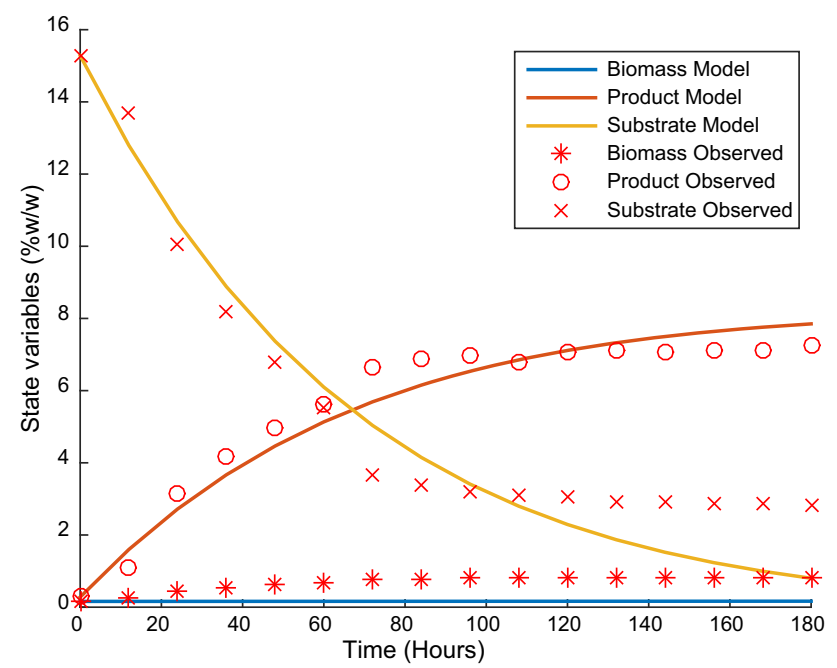

Fig. 9 Experimental results and model fitting, case of no inhibition (Monod-model)
10, 11 and 12 show comparisons between models developed for linear, sudden growth stop and exponential inhibitions during the fermentation process.

\section{Results and discussion maize extract}

Table 5 presents the parameters for the four different models used to describe the dynamics of fermentation while Figs. 1, 2, 3 and 4 presents the fitting of the models with respect to the experimental data. The results led to the following interesting conclusions; there exist ethanol inhibition in the alcoholic fermentation of maize extracts, and the patterns of inhibition could be described as linear decrease on the inhibitory ethanol concentration or sudden growth seizure at inhibitory concentration (models which showed lowest error). It is shown in this work that the errors for the models showing product inhibition were all lower than that of the Monod which was used as the control. The results confirmed previous observations by Chen and McDonald [2, 3], Beuse et al. [4], Ingledew [10], Fengwu [5], that ethanol either produced by yeast cells during fermentation or externally added into a fermentation system, can trigger inhibitions once its concentration approaches inhibitory levels. Also important in fermentation kinetics is the rate of ethanol accumulation in the fermentation broth, which depicts the maximum achievable rate of product. Even though the linear and sudden growth stop models described the dynamics of ethanol inhibition, the sudden growth stop model showed a very high maximal rate of ethanol accumulation compared to the exponential model. This suggests that designing a control policy with this model will result in high process productivity.

Tables 6 and 7 presents the model statistical validity using two sample F-test for variance and the results show that at a $99 \%$ confidence interval, the states prediction of

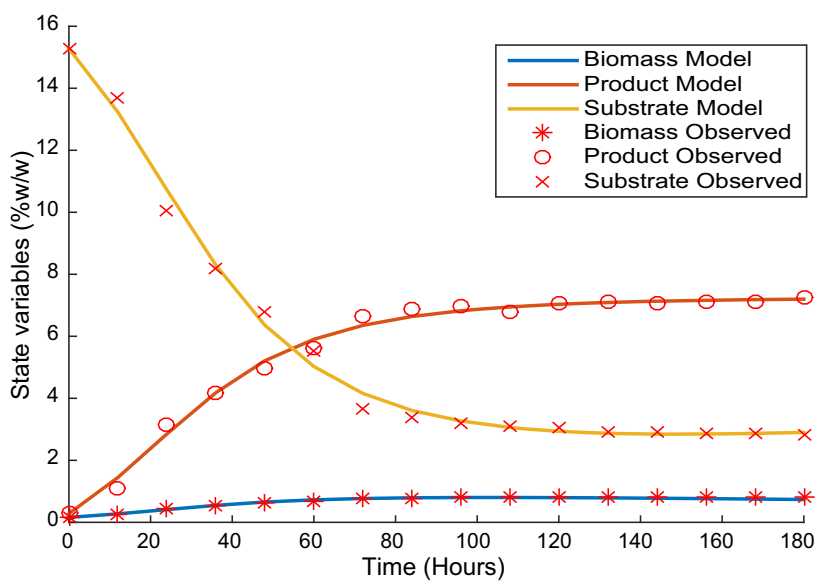

Fig. 10 Experimental results and model fitting, case of linear inhibition model 


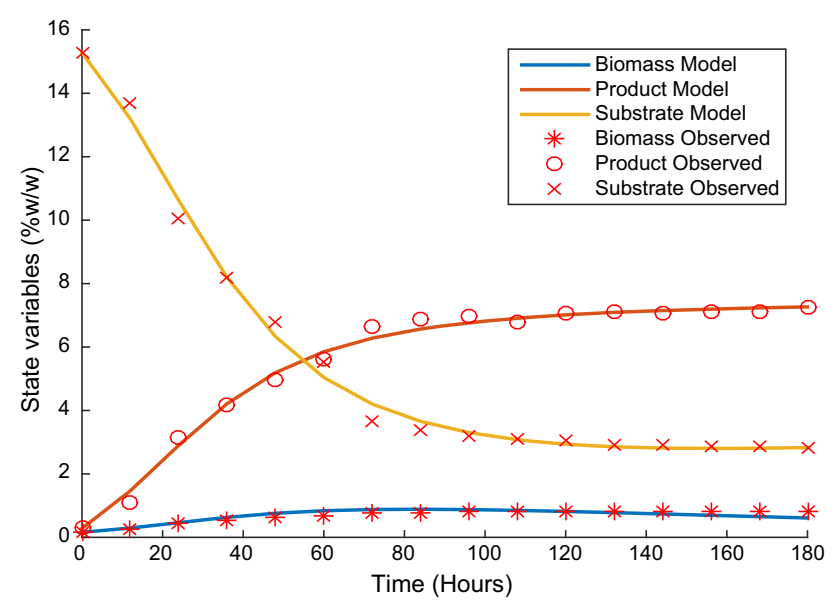

Fig. 11 Experimental results and model fitting, case of Sudden Growth Stop inhibition model

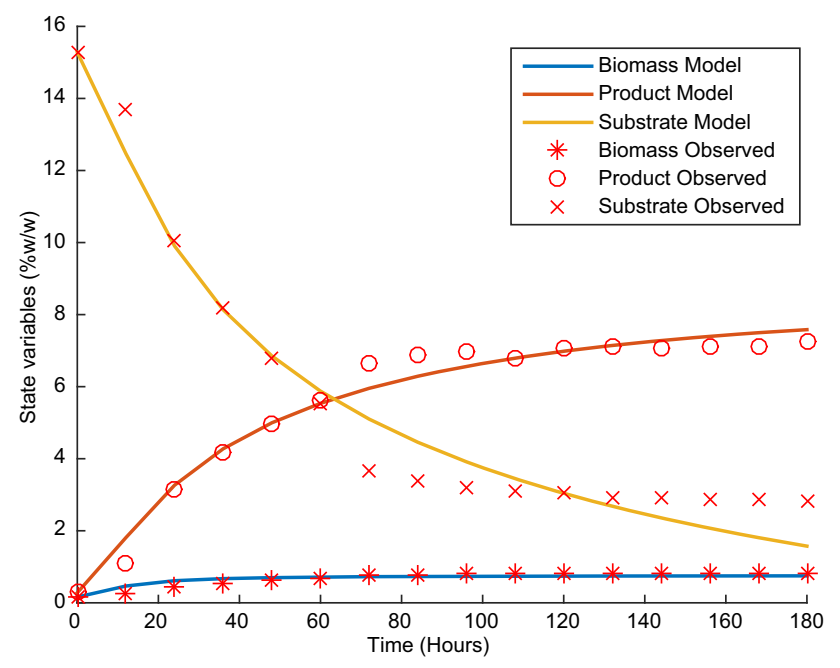

Fig. 12 Experimental results and model fitting, case of Exponential inhibition model

the linear and exponential models showed no significant difference with the experimental data.

The linear and sudden growth stop models inhibition models were both used to simulate this variation and both showed a decrease in substrate concentration and an increase in product concentration with cell growth. What was intriguing is the curve-like behavior that was observed at the end of the profiles, as shown in Figs. 13 and 14. There are two theories to the explanation of this behavior: Ethanol inhibition and stationary/decline phases in batch growth kinetics. In the case of ethanol inhibition, accumulation of ethanol to inhibitory levels leads to disruption of the cell membrane and correspondingly nonlinearity in the substrate consumption and product formation profiles, Chen and McDonald [2, 3], Beuse et al. [4], Fengwu [5], Sutton [11]. The more intense curvature observed with the sudden growth model is due to its relatively high maximal
Table 5 Model Parameters for beer fermentation using maize extracts

\begin{tabular}{|c|c|c|c|c|}
\hline & \multicolumn{4}{|l|}{ Inhibition } \\
\hline & No inhibition & Linear & SGS & Exponential \\
\hline \multicolumn{5}{|c|}{ Model parameters } \\
\hline$\mu_{\max }$ & 0.0100 & 0.0567 & 0.0630 & 2.9922 \\
\hline$q_{\text {pmax }}$ & 10.4778 & 0.7784 & 0.9338 & 8.2438 \\
\hline$P_{x \max }$ & - & - & 6.7249 & - \\
\hline$P_{p \max }$ & - & - & 7.3560 & - \\
\hline$K_{i x}$ & - & 0.1459 & - & 0.7179 \\
\hline$K_{i p}$ & - & 0.1375 & - & 0.2631 \\
\hline$K_{s x}$ & 250.000 & 1.2621 & 1.9085 & 231.9805 \\
\hline$K_{s p}$ & 200.00 & 7.1081 & 11.7803 & 199.9890 \\
\hline$Y_{x}$ & 0.1000 & 0.1084 & 0.1108 & 1.0000 \\
\hline$Y_{p}$ & 0.5370 & 1.2548 & 1.3938 & 0.6210 \\
\hline$G_{s}$ & 0.0010 & 0.0018 & 0.0051 & 0.0010 \\
\hline$M_{s}$ & 0.010 & 0.0108 & 0.0141 & 0.0100 \\
\hline Model error & 27.9231 & 2.0381 & 2.0941 & 11.340 \\
\hline
\end{tabular}

rate of product formation, hence rapidly accumulates ethanol to inhibitory concentration. Regarding the stationary and decline phase theory in batch growth kinetics, the curve-like behavior observed in the linear model can be attributed to the fact the cells grow and get to the stationary and decline phases resulting and hence no longer consume substrate and produce ethanol as in the exponential phase, resulting in the observed nonlinearity. These theories are further confirmed by the Figs. 15 and 16 which, respectively, simulates cell growth and product formation throughout the duration of fermentation. It can be observed that cells in the fermenter start declining after a certain time of fermentation buttressing the attainment of ethanol inhibition and disruption of cell membrane leading to cell death. The nonlinear patterns observed at the start of the fermentation can be attributed to high sugar concentrations encountered immediately after hydrolysis which exert osmotic stress on yeast leading to nonlinearity in their pattern of growth and product formation Russell [6], Sutton [11].

\section{Conclusions and recommendations-sorghum}

A mathematical approach to study the product inhibitor on the kinetics of alcoholic fermentation of sorghum and maize has been presented. The results of sorghum extract fermentation showed that there exist both ethanol inhibition in the alcoholic fermentation of sorghum extracts and can be described at a $99 \%$ confidence interval as being a 
Table 6 Model Statistical Validity with kinetics of linear inhibition, two sample F-test for variance

\begin{tabular}{|c|c|c|c|c|c|c|}
\hline & \multicolumn{2}{|l|}{ Biomass } & \multicolumn{2}{|l|}{ Product } & \multicolumn{2}{|l|}{ Substrate } \\
\hline & Experimental (Xobs) & Model (Xpred) & Experimental (Pobs) & Model (Ppred) & Experimental (Sobs) & Model (Spred) \\
\hline Mean & 0.673 & 0.670 & 5.580 & 5.592 & 5.644 & 5.641 \\
\hline Standard error & 0.054 & 0.052 & 0.566 & 0.556 & 1.023 & 1.017 \\
\hline Standard deviation & 0.218 & 0.206 & 2.263 & 2.223 & 4.091 & 4.069 \\
\hline Observations & 16 & 16 & 16 & 16 & 16 & 16 \\
\hline Confidence interval & 0.990 & & 0.990 & & 0.990 & \\
\hline$f$ & 0.9002 & & 0.9647 & & 0.9890 & \\
\hline $\operatorname{Pr}(F<f)$ two-tailed & 0.8414 & & 0.9455 & & 0.9832 & \\
\hline
\end{tabular}

Table 7 Model Statistical Validity with kinetics of Sudden Growth Stop inhibition, two sample $F$-test for variance

\begin{tabular}{|c|c|c|c|c|c|c|}
\hline & \multicolumn{2}{|l|}{ Biomass } & \multicolumn{2}{|l|}{ Product } & \multicolumn{2}{|l|}{ Substrate } \\
\hline & Experimental (Xobs) & Model (Xpred) & Experimental (Pobs) & Model (Ppred) & Experimental (Sobs) & Model (Spred) \\
\hline Mean & 0.673 & 0.684 & 5.579 & 5.592 & 5.644 & 5.641 \\
\hline Standard error & 0.054 & 0.052 & 0.566 & 0.555 & 1.023 & 1.016 \\
\hline Standard deviation & 0.218 & 0.209 & 2.263 & 2.221 & 4.091 & 4.066 \\
\hline Observations & 16 & 16 & 16 & 16 & 16 & 16 \\
\hline Confidence interval & 0.990 & & 0.990 & & 0.990 & \\
\hline$f$ & 0.9194 & & 0.9630 & & 0.9878 & \\
\hline $\operatorname{Pr}(\mathrm{F}<\mathrm{f})$ two-tailed & 0.8728 & & 0.9427 & & 0.9814 & \\
\hline
\end{tabular}

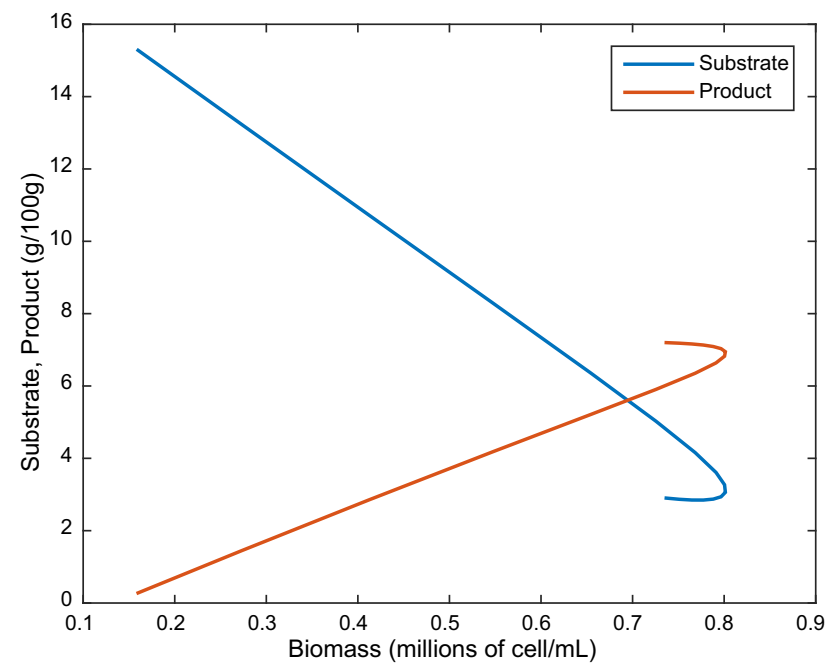

Fig. 13 Simulation of substrate and Product variation as a function of biomass during fermentation of maize extracts using Linear Inhibition kinetic model

linear and or an exponential. The results of maize extract fermentation showed that there exist ethanol inhibition which can be described with a $99 \%$ confidence interval as being either a linear or sudden growth at the inhibitory

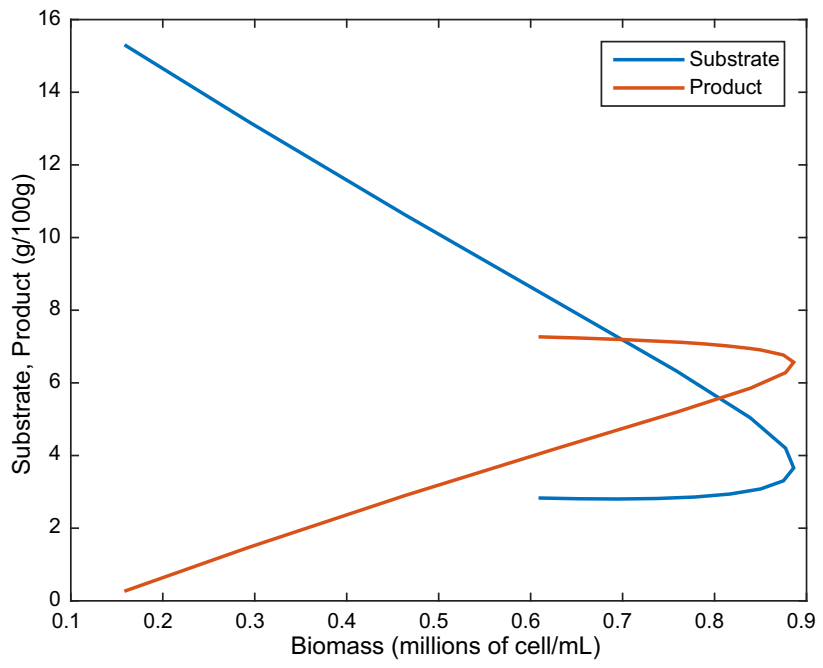

Fig. 14 Simulation of substrate and Product as a function biomass during fermentation, using Sudden Growth Stop Inhibition kinetic

ethanol concentration. The sudden growth stop model resulted in a relatively high maximal rate of ethanol accumulation hence rapidly approaching inhibitory levels. The paper, therefore, recommends for further research to study optimal control problems that can be formulated 


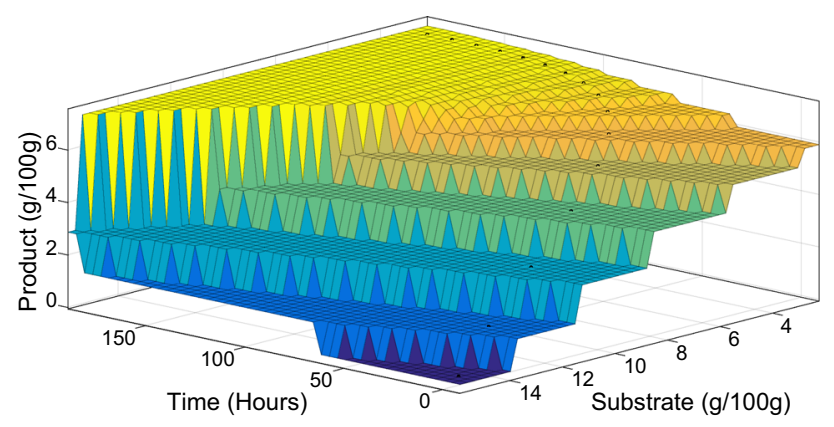

Fig. 15 3D Proximal Interpolant Simulation of product dynamics during fermentation using linear inhibition kinetic Model. Nearest neighbor interpolant

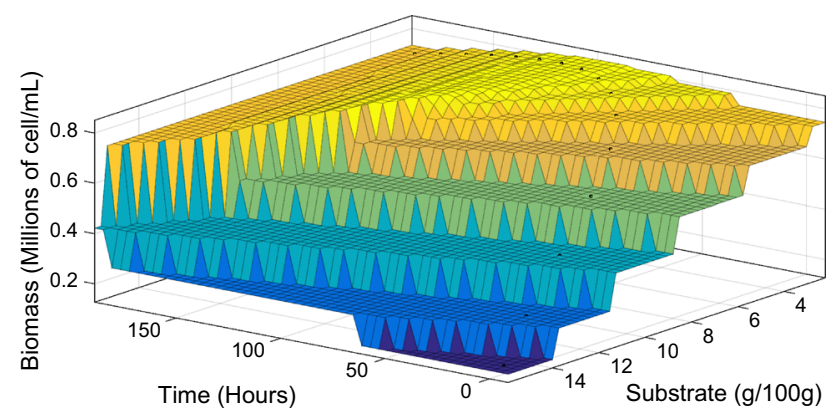

Fig. 16 3D Proximal Interpolant Simulation of Biomass Variation during fermentation using Sudden Growth Stop inhibition kinetic Model

using the models suggested in this work to understand controls variables that can minimize the effect of such inhibitions in batch fermentations.

Acknowledgements The Authors wishes to expresses their sincere appreciations to the Intra-ACP under the project "Strengthening African Higher Education through Academic Mobility", STREAM. Appreciation also goes to some breweries in Ghana for supplying us with industrial data for model validation.
Open Access This article is distributed under the terms of the Creative Commons Attribution 4.0 International License (http://crea tivecommons.org/licenses/by/4.0/), which permits unrestricted use, distribution, and reproduction in any medium, provided you give appropriate credit to the original author(s) and the source, provide a link to the Creative Commons license, and indicate if changes were made.

\section{References}

1. Alford JS (2006) Bioprocess control: advances and challenges. Comput Chem Eng 30:1464-1475

2. Chen CI, McDonald KA (1990a) Oscillatory behavior of Saccharomyces cerevisiae in continuous culture: I. Effects of $\mathrm{pH}$ and nitrogen levels. Biotechnol Bioeng 36:19-27

3. Chen CI, McDonald KA (1990b) Oscillatory behavior of Saccharomyces cerevisiae in continuous culture: II. Analysis of cell synchronization and metabolism. Biotechnol Bioeng 36:28-38

4. Beuse MRB, Kopmann AHD, Thoma M (1998) Effect of dilution rate on the mode of oscillation in continuous culture of Saccharomyces cerevisiae. J Biotechnol 61:15-31

5. Fengwu B (2007) Process Oscillations in Continuous Ethanol Fermentation with Saccharomyces cerevisiae. $\mathrm{PhD}$ thesis University of Waterloo, Ontario, Canada

6. Russell AD (2003) Similarities and differences in the responses of microorganisms to biocides. J Antimicrob Chemother 52:750-776

7. Hinshelwood C (1946) NThe chemical kinetics of the bacterial cell. Clarendon Press, Oxford

8. Ghose TK, Tyasi RD (1979) Rapid ethanol fermentation and cellulose hydrolyase. II Product and substrate inhibition and optimization of fermenter design. Biotechnol Bioeng 21(8):1401-1420

9. Aiba S, Shoda M, Nagatani M (1968) Kinetics of product inhibition in alcohol fermentation. Biotechnol Bioeng 10:845-864

10. Ingledew WM (1999) Alcohol production by saccharomyces cerevisiae: a yeast primer. In: the Alcohol Textbook. 3rd Edn, pp 49-87

11. Sutton KB (2011) Fermentation Inhibitors, Novozyme report. 18600-0 